12

\title{
Фазовый портрет рассеянного сгустка заряженных частиц
}

\author{
(c) В.Г. Куракин, ${ }^{1}$ П.В. Куракин ${ }^{2}$ \\ ${ }^{1}$ Физический институт им. П.Н. Лебедева РАН, \\ 119991 Москва, Россия \\ ${ }^{2}$ Институт проблем управления им. В.А. Трапезникова РАН, \\ 117997 Москва, Россия \\ e-mail: vgkurakin@mail.ru, erich.honeck@gmail.com
}

(Поступило в Редакцию 19 мая 2017 г. В окончательной редакции 23 октября 2017 г.)

Функция распределения для движущегося заряда, испытывающего многократное кулоновское рассеяние, использована при выводе соотношений для параметров рассеянного пучка через характеристики налетающего на мишень пучка. Введено понятие согласованного с мишенью пучка и приведена формула, связывающая поперечные эмиттансы налетающего и рассеянного пучков.

DOI: 10.21883/JTF.2018.05.45910.2342

Внутренние мишени ускорителей и накопителей заряженных частиц, перезарядные мишени, металлические фольги и диэлектрические пленки, используемые для разделения вакуумных объемов и вывода пучков из ускорителей, электрон-позитронные конверторы, мишени систем мечения - все эти устройства, широко используемые в современных установках ядерной физики, существенно влияют на характеристики прошедшего через них пучка, прежде всего через механизм многократного кулоновского рассеяния. Знание указанных характеристик необходимо для адекватного конструирования канала транспортировки прошедшего через рассеивающую среду пучка. Исследованию процессов преобразования фазового объема пучка при пересечении рассеивающей среды посвящена настоящая работа.

Постановка задачи иллюстрируется на рис. 1, на котором геометрия процесса рассеяния дополнена поперечными фазовыми портретами падающего и прошедшего через рассеивающую среду пучка заряженных частиц. Наша задача - выразить параметры эллипса, охватывающего занятыми частицами площадь в фазовом пространстве прошедшего через среду пучка через пара-

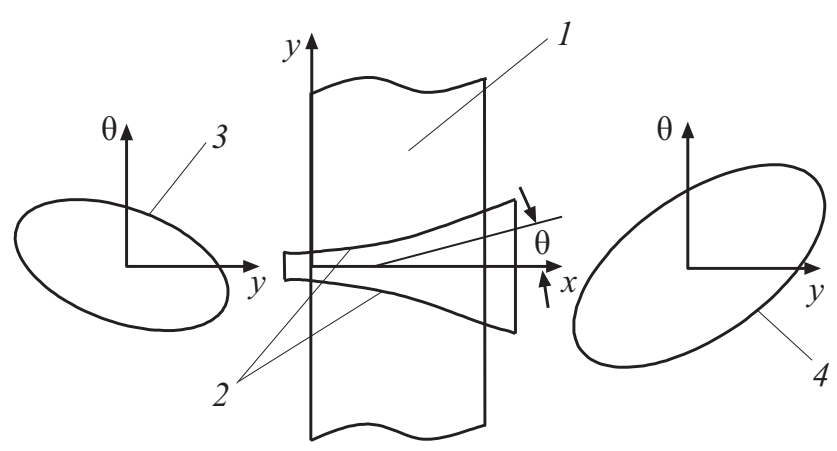

Рис. 1. Геометрия рассеяния на мишени движущегося сгустка заряженных частиц: 1 - рассеивающая среда (мишень), 2 - огибающая рассеиваемого сгустка, 3 - фазовый портрет падающего на мишень сгустка, 4 - фазовый портрет рассеянного сгустка. метры эллипса, описывающего распределение налетающего на мишень пучка.

Функция распределения для элементарного заряда, движущегося в однородной бесконечной среде и испытывающего многократное кулоновское рассеяние, имеет вид $[1,2]$ :

$$
\begin{aligned}
& P(x, y, \theta) d y d \theta=\frac{2 \sqrt{3}}{\pi} \frac{1}{\Theta_{s}^{2} x^{2}} \\
& \times \exp \left[-\frac{4}{\Theta_{s}^{2} x}\left(\theta^{2}-\frac{3 y \theta}{x}+\frac{3 y^{2}}{x^{2}}\right)\right] d y d \theta .
\end{aligned}
$$

Здесь $P(x, y, \theta) d y d \theta-$ вероятность обнаружить заряд на глубине $x$ в интервалах $(\theta, \theta+d \theta),(y, y+d y)$ углов и поперечных смещений, а

$$
\Theta_{s}^{2}=\left(\frac{E_{s}}{\beta c p}\right)^{2} \frac{1}{X_{0}}, E_{s}=\left(\frac{4 \pi}{\alpha}\right)^{1 / 2} m_{e} c^{2}=21 \mathrm{MeV},
$$

где $\beta, p, c-$ соответственно приведенная скорость заряда, его импульс и скорость света, $X_{0}$ - радиационная длина, $\alpha=\frac{e^{2}}{\hbar c}-$ постоянная тонкой структуры, $e, m_{e}-$ заряд электрона и его масса, $\hbar$ - постоянная Планка.

Согласно $(1)$, события $(y, \theta)$ равной вероятности на глубине $x$ расположены на эллипсах, описываемых в системе координат $(\eta=y / x, \theta)$ уравнением

$$
3 \eta^{2}-3 \eta \theta+\theta^{2}=f=\text { const, }
$$

где значение константы определяет относительное количество рассеиваемых частиц, охватываемых эллипсом. Среднее значение этой величины

$$
\begin{aligned}
\langle f\rangle & =\frac{2 \sqrt{3}}{\pi} \frac{1}{\Theta_{s}^{2} x} \int\left(3 \eta^{2}-3 \eta \theta+\theta^{2}\right) \\
& \times \exp \left[-\frac{4}{\Theta_{s}^{2} x}\left(3 \eta^{2}-3 \eta \theta+\theta^{2}\right)\right] d \eta d \theta=\frac{1}{4} \Theta_{s}^{2} x,
\end{aligned}
$$

определяет эллипс, который естественно принять за фазовый портрет пучка, имеющего нитеобразную форму на 
входе в рассеивающую среду (поперечное распределение в виде $\delta$-функции), а площадь эллипса рассматривать как эмиттанс, нормированный на толщину рассеивающей пластины

$$
S=\frac{2 \pi\langle f\rangle}{\sqrt{3}}=\frac{\pi}{2 \sqrt{3}} \Theta_{s}^{2} x .
$$

В более привычных в оптике пучков единицах измерения - радианы и сантиметры - площадь эллипса рассеяния равна

$$
\varepsilon=\frac{\pi}{2 \sqrt{3}} \Theta_{s}^{2} x^{2}=\frac{\pi}{2 \sqrt{3}}\left(\frac{E_{s}}{\beta c p}\right)^{2} \frac{x^{2}}{X_{0}} .
$$

В конкретных расчетах, требующих учета как можно большего числа, приближающегося к 100\%, рассеянных частиц, следует использовать примерно на порядок большую величину, что приблизительно соответствует правилу $3 \sigma$ для одномерных нормальных распределений.

Для заряда, падающего на мишень толщиной $h$ в точке $Y$ под углом $\Theta \ll 1$, имеет место следующее распределение вероятностей:

$$
\begin{aligned}
& P(Y, \Theta, y, \theta)=\frac{2 \sqrt{3}}{\pi} \frac{1}{\Theta_{s}^{2} h^{2}} \\
& \times\left\{-\frac{4}{\Theta_{s}^{2}}\left[\frac{(\theta-\Theta)^{2}}{h}-\frac{3(y-Y-h \Theta)(\theta-\Theta)}{h^{2}}\right.\right. \\
& \left.\left.+\frac{3(y-Y-h \Theta)^{2}}{h^{3}}\right]\right\} .
\end{aligned}
$$

Из последнего соотношения следует, что каждой точке фазового портрета налетающего пучка $Y, \Theta$ соответствует элементарный эллипс рассеяния с центром в точке $(Y+h \Theta, \Theta)$ фазовой плоскости $(y, \theta)$, а граница фазового портрета рассеянного пучка определяется огибающей к семейству эллипсов рассеяния с центрами, лежащими на эллипсе, описываемом уравнением

$$
A_{1}(\eta-\theta)^{2}+A_{2}(\eta-\theta) \theta+A_{3} \theta^{2}=F .
$$

Здесь коэффициенты $A_{1}, A_{2}, A_{3}, F$ описывают фазовый эллипс падающего на мишень пучка

$$
A_{1} \eta^{2}+A_{2} \eta \theta+A_{3} \theta^{2}=F .
$$

Площадь, охватываемая огибающей, определяет эмиттанс рассеянного в мишени пучка. Для его вычисления перейдем к новой системе координат, в которой эллипс рассеяния имеет форму окружности с радиусом, равным малой полуоси эллипса. В этой системе координат $(\mu, v)$ эллипс (9) описывается уравнением

$$
B_{1} \mu^{2}+B_{2} \mu v+B_{3} v^{2}=F
$$

коэффициенты которого $B_{i}, i=1,2,3$ вычисляются как

$$
B=M_{3} M_{2} M_{1} A \text {. }
$$

Здесь $A$ и $B$ - матрицы-столбцы, составленные из коэффициентов $A_{i}, B_{i} . M_{1}, M_{2}, M_{3}$ - квадратные матрицы третьего порядка:

$$
\begin{gathered}
M_{1}=\left[\begin{array}{ccc}
1 & 0 & 0 \\
-2 & 1 & 0 \\
1 & -1 & 1
\end{array}\right], \\
M_{2}=\left[\begin{array}{ccc}
\frac{1}{2}-\frac{1}{\sqrt{13}} & \frac{3}{2 \sqrt{13}} & \frac{1}{2}+\frac{1}{\sqrt{13}} \\
-\frac{3}{\sqrt{13}} & -\frac{2}{\sqrt{13}} & \frac{3}{\sqrt{13}} \\
\frac{1}{2}+\frac{1}{\sqrt{13}} & -\frac{3}{2 \sqrt{13}} & \frac{1}{2}-\frac{1}{\sqrt{13}},
\end{array}\right], \\
M_{3}=\left[\begin{array}{ccc}
k^{2} & 0 & 0 \\
0 & k & 0 \\
0 & 0 & 1
\end{array}\right],
\end{gathered}
$$

где $k=(4+\sqrt{13}) / \sqrt{3} \approx 4.4-$ отношение полуосей эллипса рассеяния. Смысл преобразования (11) очевиден. Первая матрица - это матричная форма записи коэффициентов уравнения (8) через коэффициенты эллипса (9). Вторая матрица осуществляет преобразование коэффициентов эллипса (8) при переходе к новой системе координат, в которой эллипс рассеяния канонический. Эта система координат получена вращением против часовой стрелки системы координат $(\eta, \theta)$ на угол $\psi$, которой равен углу наклона большой полуоси эллипса рассеяния (3) к оси $\eta$ и, согласно $(3), \operatorname{tg}(2 \psi)=-1.5$. Третья матрица осуществляет преобразование коэффициентов эллипса при сжатии в $k$ раз плоскости, где эллипс рассеяния канонический, в направлении большой полуоси эллипса рассеяния, совпадающей с горизонтальной осью $\mu$. После такого сжатия эллипс рассеяния превращается в окружность с радиусом, равным его малой полуоси.

В системе координат $(\mu, v)$ фазовый портрет рассеянного пучка определяется огибающей к семейству окружностей с центрами на эллипсе (10) и радиусами, равными малой полуоси эллипса рассеяния $b=\sqrt{S / \pi k}$, а площадь данной фигуры равна

$$
S_{1}^{\prime}=S_{0}^{\prime}+\pi b^{2}+b L^{\prime}
$$

Здесь $S_{0}^{\prime}, L^{\prime}-$ соответственно площадь и периметр эллипса (10). Учитывая, что $\pi b^{2}=S^{\prime}$, где $S^{\prime}-$ площадь эллипса рассеяния в системе координат $(\mu, v)$, получим из последнего равенства, что $S_{1}^{\prime}$ принимает минимальное значение при минимальном периметре преобразованного первоначального фазового портрета пучка. При одной и той же площади фигуры периметр минимален, когда фигура имеет форму окружности. Таким образом, минимальное значение эмиттанса рассеянного пучка имеет место тогда, когда в системе координат $\mu, v$ фазовый портрет падающего на мишень пучка имеет, как и эллипс рассеяния, форму окружности. В этом случае фазовый портрет пучка, преобразованный матрицей $M_{1}$ 

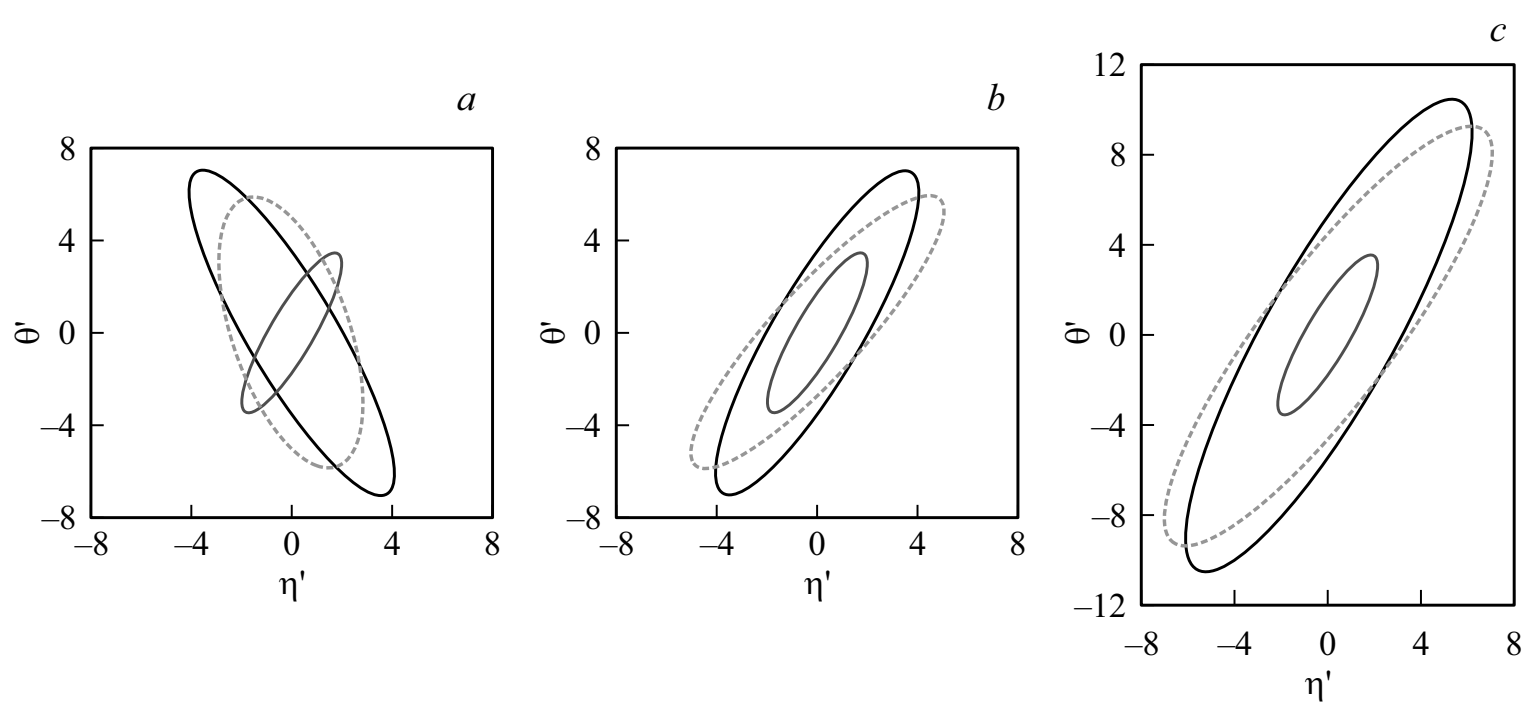

Рис. 2. Пример определения фазового портрета рассеянного на мишени сгустка заряженных частиц. Показаны фазовые портреты: $a$ - падающих согласованного и произвольного сгустков (пунктир) на фоне фазового портрета рассеянного игольчатого пучка (малый эллипс), $b-$ после преобразования прямолинейного промежутка, $c-$ рассеянных сгустков. Используются единицы $\theta^{\prime}=2 \sqrt{3} \theta / \Theta_{s} \sqrt{x}, \eta^{\prime}=2 \sqrt{3} \eta / \Theta_{s} \sqrt{x}$

(соответствующей прямолинейному промежутку), подобен эллипсу рассеяния (3), а фазовый портрет налетающего пучка, согласно $(8,9)$, в системе координат $(\eta, \theta)$ описывается эллипсом

$$
3 \eta^{2}+3 \eta \theta+\theta^{2}=F_{1} .
$$

Будем называть такой пучок согласованным с рассеивающей средой. Так как в случае согласованного пучка $L^{\prime}=2 \pi R=2 \pi \sqrt{S_{0}^{\prime} / \pi}=2 \sqrt{\pi S_{0}^{\prime}}, b=\sqrt{S^{\prime} / \pi}$, соотношение (13) преобразуется в

$$
S_{1}^{\prime}=S_{0}^{\prime}+2 \sqrt{S_{0}^{\prime} S^{\prime}}+S^{\prime}
$$

Поскольку преобразование фазовой плоскости, осуществляемое матрицами $M_{1}, M_{2}$, не изменяет площади фигур, а преобразование $M_{3}$ уменьшает их в $k$ раз, то аналогичное соотношение для согласованного пучка, которое перепишем в более компактном виде, имеет место и в исходной системе координат:

$$
\sqrt{S_{1}}=\sqrt{S_{0}}+\sqrt{S}
$$

В общем случае несогласованного пучка вычисление эмитанса рассеянного пучка сводится к вычислению эллиптического интеграла, выражаемого через параметры преобразованного эллипса и умноженного на коэффициент $k$. Заметим, что фазовый портрет рассеянного пучка описывается кривой более высокого порядка, чем второй, что не совсем удобно для приложений в оптике пучков заряженных частиц. Поэтому аппроксимируем его границу эллипсом с той же ориентацией осей как у (10) и полуосями, увеличенными на малую полуось эллипса рассеяния:

$$
S_{1}=k \pi(\alpha+b)(\beta+b)=S_{0}+S+\sqrt{S \pi}(\alpha+\beta),
$$

где полуоси $\alpha, \beta$ определяются из коэффициентов $B_{i}, F$, описывающих эллипс (10):

$$
\begin{gathered}
\alpha=\sqrt{\frac{2 F\left(\sqrt{\left(B_{1}-B_{3}\right)^{2}+B_{2}^{2}}+B_{1}+B_{3}\right)}{4 B_{1} B_{3}-B_{2}^{2}}}, \\
\beta=\sqrt{\frac{2 F}{\sqrt{\left(B_{1}-B_{3}\right)^{2}+B_{2}^{2}}+B_{1}+B_{3}}}
\end{gathered}
$$

Коэффициенты, описывающие аппроксимирующий эллипс рассеянного пучка, определяются согласно соответствующим формулам аналитической геометрии

$$
\begin{gathered}
C_{1}^{\prime}=(\alpha+b)^{2} \frac{\operatorname{tg}^{2} \varphi}{1+\operatorname{tg}^{2} \varphi}+(\beta+b)^{2} \frac{1}{1+\operatorname{tg}^{2} \varphi}, \\
C_{2}^{\prime}=2\left[(\beta+b)^{2}-(\alpha+b)^{2}\right] \frac{\operatorname{tg} \varphi}{1+\operatorname{tg}^{2} \varphi}, \\
C_{3}^{\prime}=(\beta+b)^{2} \frac{\operatorname{tg}^{2} \varphi}{1+\operatorname{tg}^{2} \varphi}+(\alpha+b)^{2} \frac{1}{1+\operatorname{tg}^{2} \varphi}, \\
\operatorname{tg} \varphi=\frac{B_{3}-B_{1} \pm \sqrt{\left(B_{3}-B_{1}\right)^{2}+B_{2}^{2}}}{B_{2}}, \\
F_{c}=(\alpha+b)^{2}(\beta+b)^{2} .
\end{gathered}
$$

В исходной системе координат коэффициенты эллипca, аппроксимирующего фазовый портрет рассеянного пучка, определяются применением обратного преобразования к вектору-столбцу $C^{\prime}$ и имеют вид

$$
C=M_{2}^{-1} M_{3}^{-1} C^{\prime}
$$


Рис. 2 демонстрирует описанную выше технику вычисления эмиттанса рассеянного пучка и коэффициентов эллипса, описывающего фазовый портрет последнего согласно приведенным алгоритмам. Используются единицы $\theta^{\prime}=2 \sqrt{3} \theta / \Theta_{s} \sqrt{x}, \eta^{\prime}=2 \sqrt{3} \eta / \Theta_{x} \sqrt{x}$. Матрица $M_{1}$ в оптике пучков заряженных частиц соответствует матрице свободного промежутка, равного толщине рассеивающей среды. Огибающая к семейству эллипсов рассеяния, центры которых расположены вдоль границы преобразованного эллипса, образует границу фазового портрета пучка после прохождения рассеивающей среды. Альтернативный метод - решение дифференциального уравнения огибающей с последующим вычислением площади, охватываемой этой огибающей - не позволяет получить аналитические выражения. Он также не позволяет получить аналитическое представление аппроксимирующего эллиптического портрета рассеянного пучка. Заметим, что параметр $\Theta_{s}(2)$ определяет параметры материала мишени и пучка. Так для алюминия (радиационная длина $X_{0} \approx 26 \mathrm{~g} / \mathrm{cm}^{2} \approx 10 \mathrm{~cm}$ ) и электрона с энергией $21 \mathrm{MeV} \Theta_{s}^{2} \approx 0.11 / \mathrm{cm}$, для меди $\left(X_{0} \approx 13 \mathrm{~g} / \mathrm{cm}^{2} \approx 1.4 \mathrm{~cm}\right)$ для электрона с той же энергией $\Theta_{s}^{2} \approx 0.71 / \mathrm{cm}$.

Представленный и описанный выше способ определения параметров эллипса, описывающего фазовый портрет рассеянного на мишени пучка, основан на использовании функции распределения и преобразовании фазовой плоскости поперечная координата-угол методами аналитической геометрии. Такой подход представляется наиболее адекватным, поскольку нацелен на использование результатов для решения задач оптики пучков заряженных частиц в самых различных постановках задач. Известно, что многие широко используемые программные коды (например, [3]) основаны на описании пучка через параметры многомерного эллипсоида в фазовом пространстве, и описанное выше преобразование фазового портрета пучка в рассеивающей среде может напрямую использоваться с небольшими модификациями в данных кодах. Необходимо также отметить и другое не менее важное обстоятельство. Электромагнитные элементы электронной оптики не изменяют фазовый объем пучка заряженных частиц (теорема Лиувилля), и при использовании рассеивающих мишеней в каналах изменение эмиттанса происходит локально в месте расположения мишени. Развитый нами аппарат и отражает это. При альтернативном подходе (например [4]), где прослеживаются траектории отдельных частиц, а учет мишени сводится к стохастическому процессу изменения поперечного импульса заряда, процесс увеличения эмиттанса „замазывается“ магнитной оптикой. При описанном в нашей работе подходе процесс оптимизации оптики с целью минимизации эмиттанса пучка физически понятен и нагляден, и поэтому наиболее быстро ведет к цели.

\section{Список литературы}

[1] Росси Б. Частицы больших энергий / Пер. с англ. М.: ГИТТЛ, $1955.536 \mathrm{c.}$

[2] Беленький С.3. Лавинные процессы в космических лучах. М.: ГИТТЛ, 1948. 244 c.

[3] Brown K.L., Carey D.C., Iselin Ch., Rothacker F. TRANSPORT, a computer program for desining charged particle beam transport systems. Geneva, 1980. $251 \mathrm{p}$.

[4] Hinterberger F., Prasuhn D. // Nucl. Instrum. Method. Phys. Res., A. 1989. Vol. 279. P. 413-422. 